OPEN ACCESS

Edited by: Mark Girgis,

University of California, Los Angeles, United States

Reviewed by: Gianni Mura,

Ospedale del Valdarno, Italy Antonio Sommariva, Veneto Institute of Oncology (IRCCS),

*Correspondence: Dong Peng carry_dong@126.com

tThese authors have contributed equally to this work

Specialty section: This article was submitted to Surgical Oncology, a section of the journal Frontiers in Oncology

Received: 26 May 2021 Accepted: 31 July 2021 Published: 24 August 2021

Citation:

Tao W, Liu X-Y, Cheng Y-X, Kang B,

Zhang $H$, Yuan $C$, Zhang $B$ and

Peng D (2021) Does Extended Intraoperative Peritoneal Lavage Really Bring Benefit on Patients With Gastric

Cancer? A Meta-Analysis of Published Clinical Trials.

Front. Oncol. 11:715040. doi: 10.3389/fonc.2021.715040

\section{Does Extended Intraoperative Peritoneal Lavage Really Bring Benefit on Patients With Gastric Cancer? A Meta-Analysis of Published Clinical Trials}

\author{
Wei Tao ${ }^{1 \dagger}$, Xiao-Yu Liu ${ }^{1 \dagger}$, Yu-Xi Cheng ${ }^{1}$, Bing Kang ${ }^{2}$, Hua Zhang ${ }^{1}$, Chao Yuan ${ }^{1}$, \\ Bin Zhang ${ }^{1}$ and Dong Peng ${ }^{1 *}$ \\ 1 Department of Gastrointestinal Surgery, The First Affiliated Hospital of Chongqing Medical University, Chongqing, China, \\ ${ }^{2}$ Department of Clinical Nutrition, The First Affiliated Hospital of Chongqing Medical University, Chongqing, China
}

Purpose: The purpose of the current meta-analysis is to analyze whether extended intraoperative peritoneal lavage (EIPL) can bring benefit on short-term outcomes or survival for patients undergoing curative gastrectomy for gastric cancer.

Methods: The PubMed, Embase, and Cochrane Library databases were searched from inception to May 3, 2021, to find eligible studies. Postoperative complications, overall survival (OS), disease-free survival (DFS), and peritoneal recurrence-free survival (PRFS) were compared between EIPL group and No EIPL group.

Results: A total of five randomized controlled trials with 1,790 patients were included in the current meta-analysis. No difference was found in baseline information ( $p>0.05)$. After pooling up the data of overall postoperative complications, no significant difference was found between EIPL group and No EIPL group $(\mathrm{OR}=0.88,95 \% \mathrm{Cl}=0.51$ to $1.53, \mathrm{P}=$ 0.65). Furthermore, there was no significant difference between EIPL group and No EIPL group in terms of $\mathrm{OS}(\mathrm{HR}=0.77,95 \% \mathrm{Cl}=0.36$ to $1.64, \mathrm{P}=0.49)$, DFS $(\mathrm{HR}=0.97,95 \%$ $\mathrm{Cl}=0.71$ to $1.33, \mathrm{P}=0.87)$, and PRFS $(\mathrm{HR}=1.03,95 \% \mathrm{Cl}=0.74$ to $1.43, \mathrm{P}=0.86)$. In terms of subgroup analysis of OS, no significant difference was found as well $(H R=1.05$, $95 \% \mathrm{Cl}=0.82$ to $1.34, \mathrm{P}=0.69$ ).

Conclusions: EIPL did not bring benefit in terms of short-term outcomes or survival. Therefore, EIPL is not recommended for patients undergoing curative gastrectomy for gastric cancer.

Keywords: extended intraoperative peritoneal lavage, gastric cancer, meta-analysis, outcomes, overall survival 


\section{INTRODUCTION}

Gastric cancer is the fifth most common cancer in the world and the third leading cause of cancer-related deaths, especially in East Asia (1), and gastrectomy is still the main treatment for gastric cancer (2-4). Peritoneal metastasis (PM) is the main method of distant metastasis in gastric cancer and the main cause of cancerrelated mortality. PM is detected in $10-30 \%$ of patients with gastric cancer at the time of initial diagnosis $(5,6)$, and furthermore, more than $50 \%$ of patients with stage II-III tumors develop PM within 5 years after gastrectomy $(7,8)$. Once PM occurs, symptoms such as refractory peritoneal effusion and cachexia may appear, which are the main causes of death (9).

The occurrence of PM may be caused by free cancer cells shed from the surface of the gastric serous membrane $(10,11)$. In addition, the operation of gastrectomy or lymph node dissection may cause cancer cells to fall off $(12,13)$. The elimination of free cancer cells during gastrectomy may effectively reduce the peritoneal recurrence of gastric cancer. Extensive intraoperative peritoneal lavage (EIPL) is a treatment method for preventing free cancer cells. The procedure of EIPL is as follows: the abdominal cavity is repeatedly flushed with $1 \mathrm{~L}$ of normal saline (up to 10 times) after gastrectomy $(14,15)$.

However, the complications and survival of EIPL treatment of gastric cancer are controversial. Previous studies have reported that EIPL could prolong overall survival and reduce complications $(16,17)$. However, other studies have shown that EIPL does not bring significant survival benefits and complications (18-20). Therefore, the purpose of the current meta-analysis is to analyze whether EIPL can bring benefit on short-term outcomes or survival.

\section{METHODS}

This meta-analysis was conducted in accordance with the Preferred Reporting Items for Systematic Reviews and MetaAnalyses (PRISMA) statement (21).

\section{Literature Search Strategy}

The PubMed, Embase, and Cochrane Library databases were searched from inception to May 3, 2021, to find eligible studies. There were two key items, namely, extended intraoperative peritoneal lavage and gastric cancer. The search strategy for extended intraoperative peritoneal lavage was as follows: "extended intraoperative peritoneal lavage" OR "EIPL". The search strategy for gastric cancer was as follows: "gastric cancer" OR "gastric carcinoma" OR "gastric neoplasms" OR "stomach cancer" OR "stomach carcinoma" OR "stomach neoplasms." Then, we combined the two key search items using "AND"; moreover, the language was limited in English.

\section{Inclusion and Exclusion Criteria}

The inclusion criteria were as follows: (1) patients who underwent EIPL and standard gastrectomy for gastric cancer;
(2) the EIPL and No EIPL treatments were both reported; and (3) there was at least one outcome reported including postoperative complications and survival analysis. The exclusion criteria were as follows: (1) letters, comments, reviews, conferences, or case reports; and (2) insufficient data for extraction.

The inclusion and exclusion were conducted by two reviewers, respectively. Disagreement was settled by group discussion.

\section{Study Selection}

The databases were searched by two reviewers independently. The titles and abstracts were screened after duplicate records were removed. After that, full texts were evaluated according to inclusion and exclusion criteria. Two reviewers conducted the study selection, and if disagreement occurred, final judgment was made by group discussion.

\section{Data Extraction}

The data were extracted and cross-checked by two reviewers. The extracted data were as follows: the first author, publication year, country, study design, sample size, baseline information, surgical information, postoperative complications, and survival information.

\section{Outcomes and Definition}

The primary outcome of the current meta-analysis was the survival analyses, which included overall survival (OS), diseasefree survival (DFS), and peritoneal recurrence-free survival (PRFS). The secondary outcome was the postoperative complications, which included anastomotic leakage, pancreatic fistula, abdominal abscess, wound problems, postoperative bleeding, and short-term death.

The classification of postoperative complications was according to the Clavien-Dindo classification (22). OS was defined as the time from diagnosis to death from any cause. DFS was defined as the time from diagnosis to the time of recurrence, death, or last follow-up. PRFS was defined as the time from diagnosis to the time of peritoneal recurrence, death, or last follow-up.

\section{Quality Assessment}

The criteria described in the Cochrane Handbook for Systematic Reviews of Interventions were used for evaluating the risk of bias in the included studies (23). Two reviewers conducted the bias evaluation, respectively.

\section{Statistical Analysis}

In the current meta-analysis, continuous variables are presented as the mean and standard deviation (SD), and categorical variables are presented as proportions. For dichotomous and continuous variables, odds ratios (ORs) and mean differences (MDs) were calculated, and 95\% confidence intervals (CIs) were calculated. The pooled hazard ratios (HRs) and the $95 \%$ confidence intervals (CIs) of each study were calculated to estimate the survival outcome. The $\mathrm{I}^{2}$ value and the results of the chi-squared test were used to assess the statistical heterogeneity $(24,25)$. High heterogeneity was considered when $\mathrm{I}^{2}>50 \%$; in such cases, the random effects model was used, and $\mathrm{p}<0.1$ was considered statistically significant. The fixed effects model was used when $\mathrm{I}^{2} \leq$ 
$50 \%$, and $\mathrm{p}<0.05$ was considered statistically significant. This meta-analysis was performed with RevMan 5.3 (The Cochrane Collaboration, London, United Kingdom).

\section{RESULTS}

\section{Study Selection}

A total of 58 studies (21 studies in PubMed, 27 studies in Embase, and 10 studies in the Cochrane library) were identified in the databases. Forty studies were left after removing the duplicates. Fifteen studies were accessed by fulltext scanning, and finally, five randomized controlled trials (RCTs) were included (Figure 1).

\section{Characteristics of the Included Studies and Risk Bias Assessment}

A total of five studies (16-20) with 1,790 patients were included in the current meta-analysis. The publication year was from 2009 to 2021. Four RCTs were from Asia, and one RCT was from Spain. The detailed information about sample size and intervention method is shown in Table 1. Risk bias was accessed through seven aspects of the study, namely, random sequence generation, allocation concealment, blinding of the participants and personnel, selective reporting, incomplete outcome data, blinding of the outcome assessment, and other biases. The risk of bias summary and risk of bias graph are shown in Figure 2.

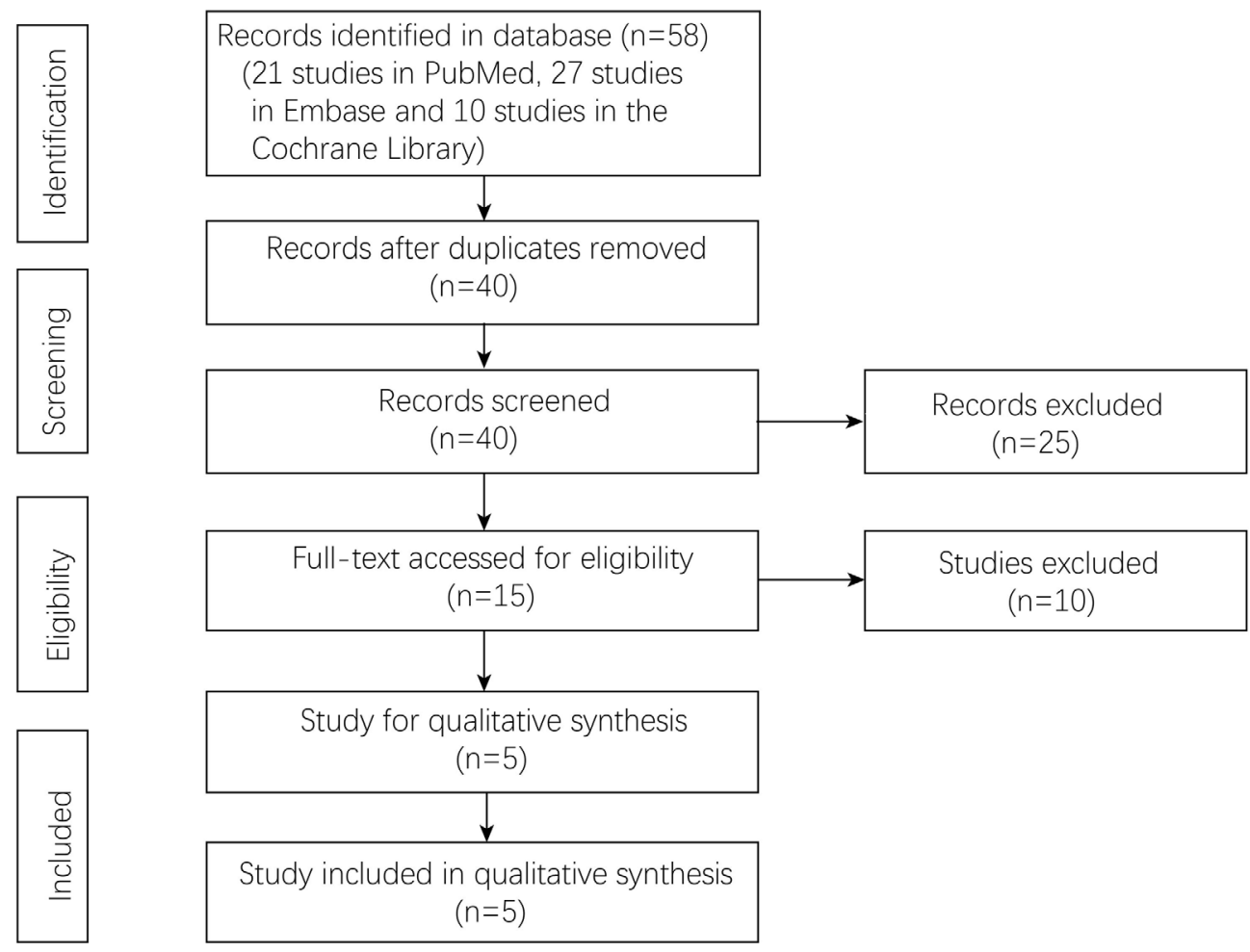

FIGURE 1 | Flowchart of study selection.

TABLE 1 | Characteristics of the included studies.

\begin{tabular}{|c|c|c|c|c|c|c|c|c|}
\hline Author & Year & Country & Study design & \multicolumn{2}{|c|}{ Method } & \multicolumn{3}{|c|}{ Sample size } \\
\hline Misawa K & 2019 & Japan & $\mathrm{RCT}$ & Surgery+ EIPL & Surgery & 145 & 150 & 295 \\
\hline Rodríguez-Santiago J & 2021 & Spain & $\mathrm{RCT}$ & Surgery+ EIPL & Surgery & 43 & 43 & 86 \\
\hline Kuramoto M & 2009 & Japan & $\mathrm{RCT}$ & Surgery+ EIPL+ IPC & Surgery & 30 & 29 & 59 \\
\hline Yang HK & 2021 & Singapore, Malaysia, Korea, China, and Japan & $\mathrm{RCT}$ & Surgery+ EIPL & Surgery & 398 & 402 & 800 \\
\hline
\end{tabular}

EIPL, extended intraoperative peritoneal lavage; $R C T$, randomized controlled trials; IPC, intraperitoneal chemotherapy. 

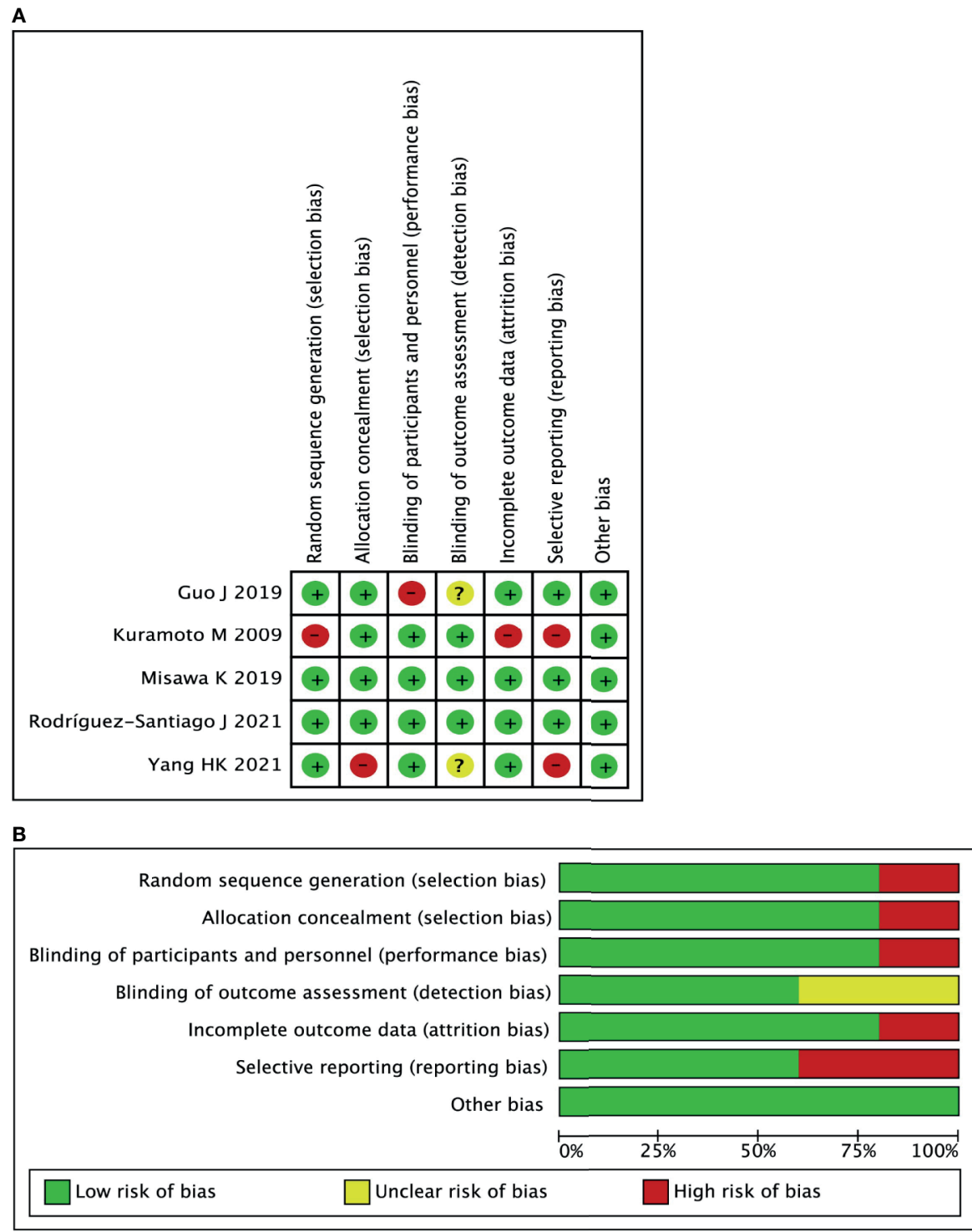

FIGURE 2 | Risk of bias for each included study. (A) risk of bias summary. (B) Risk of bias graph.

\section{Summary of Information Between EIPL Group and No EIPL Group}

The baseline information including age, sex, body mass index (BMI), American Society of Anesthesiologists (ASA), T and N stage were compared between EIPL group and No EIPL group, and no significant difference was found ( $p>0.05)$. There was no significant difference in terms of surgical information including gastrectomy method, reconstruction method, and combined organ resection $(\mathrm{p}>0.05)$ (Table 2$)$.

\section{Comparison of Complications Between EIPL Group and No EIPL Group}

Data regarding overall postoperative complications were extracted from four studies $(16,18-20)$. After pooling up the data, no significant difference was found between EIPL group and
No EIPL group $(\mathrm{OR}=0.88,95 \% \mathrm{CI}=0.51$ to $1.53, \mathrm{P}=0.65)$ (Figure 3A). In the subgroup meta-analysis of $\geq$ grade III complications, there was no significant difference between EIPL group and No EIPL group $(\mathrm{OR}=0.44,95 \% \mathrm{CI}=0.04$ to 4.37 , $\mathrm{P}=0.48$ ) (Figure 3B). The detailed postoperative complications including anastomotic leakage, pancreatic fistula, abdominal abscess, wound problems, postoperative bleeding, and shortterm death were pooled up, and no significant difference was found between EIPL group and No EIPL group ( $p>0.05)$.

\section{Survival Analysis Between EIPL Group and No EIPL Group}

There were four studies reporting OS (17-20) and two studies reporting DFS and PRFS $(18,19)$. After pooling up all the data, no significant difference was found between EIPL group and No 
TABLE 2 | Summary of information between EIPL group and No EIPL group.

\begin{tabular}{|c|c|c|c|c|}
\hline Characteristics & Studies & Participants (EIPL / No EIPL) & Mean Difference / Odds Ratio (95\% Cl) & Heterogeneity \\
\hline \multicolumn{5}{|l|}{ Baseline information } \\
\hline Age, year & 4 & $497 / 493$ & $-0.03[-1.29,1.23] ; P=0.97$ & $\mathrm{I}^{2}=0 \% ; P=0.79$ \\
\hline Male & 5 & $895 / 895$ & $0.98[0.80,1.20] ; P=0.88$ & $\mathrm{I}^{2}=0 \% ; P=0.67$ \\
\hline $\mathrm{BMl}, \mathrm{kg} / \mathrm{m}^{2}$ & 2 & $424 / 421$ & $0.13[-0.30,0.56] ; P=0.56$ & $\mathrm{I}^{2}=0 \% ; P=0.66$ \\
\hline ASA 1-2 & 2 & $440 / 444$ & $1.14[0.72,1.80] ; P=0.58$ & $\mathrm{I}^{2}=28 \% ; P=0.24$ \\
\hline ASA 3-4 & 2 & $440 / 444$ & $0.88[0.56,1.39] ; P=0.58$ & $\mathrm{I}^{2}=28 \% ; P=0.24$ \\
\hline T1-T3 & 4 & $862 / 864$ & $0.95[0.78,1.16] ; P=0.61$ & $\mathrm{I}^{2}=0 \% ; P=0.99$ \\
\hline T4 & 4 & $862 / 864$ & $1.05[0.86,1.28] ; P=0.61$ & $\mathrm{I}^{2}=0 \% ; P=0.99$ \\
\hline NO & 4 & $865 / 866$ & $0.94[0.76,1.18] ; P=0.61$ & $\mathrm{I}^{2}=0 \% ; P=0.88$ \\
\hline $\mathrm{N} 1-\mathrm{N} 3$ & 4 & $865 / 866$ & $1.06[0.85,1.32] ; P=0.61$ & $1^{2}=0 \% ; P=0.88$ \\
\hline \multicolumn{5}{|l|}{ Surgical information } \\
\hline Total gastrectomy & 5 & $895 / 895$ & $1.07[0.88,1.29] ; P=0.50$ & $\mathrm{I}^{2}=29 \% ; P=0.23$ \\
\hline Roux-en-Y & 2 & $441 / 445$ & $1.12[0.85,1.48] ; P=0.42$ & $I^{2}=0 \% ; P=0.85$ \\
\hline Combined organ resection & 3 & $467 / 464$ & $1.02[0.69,1.50] ; P=0.92$ & $I^{2}=36 \% ; P=0.21$ \\
\hline \multicolumn{5}{|l|}{ Postoperative complications } \\
\hline Anastomotic leakage & 4 & $813 / 826$ & $1.33[0.65,2.71] ; P=0.43$ & $\mathrm{I}^{2}=0 \% ; P=0.79$ \\
\hline Pancreatic fistula & 4 & $813 / 826$ & $0.51[0.23,1.13] ; P=0.10$ & $I^{2}=0 \% ; P=0.83$ \\
\hline Abdominal abscess & 4 & $813 / 826$ & $0.87[0.45,1.66] ; P=0.67$ & $\mathrm{I}^{2}=29 \% ; P=0.24$ \\
\hline Wound problems & 2 & $625 / 633$ & $1.51[0.61,3.69] ; P=0.37$ & $\mathrm{I}^{2}=75 \% ; P=0.05$ \\
\hline Postoperative bleeding & 2 & $625 / 633$ & $0.70[0.30,1.64] ; P=0.41$ & $1^{2}=7 \% ; P=0.30$ \\
\hline Short-term death & 2 & $424 / 421$ & $0.12[0.02,0.98] ; P=0.05$ & $I^{2}=0 \% ; P=0.69$ \\
\hline
\end{tabular}

EIPL, extended intraoperative peritoneal lavage; BMI, body mass index; ASA, American Society of Anesthesiologists; T, Tumor depth; N, lymph nodes.

Postoperative complications (Grade $\geq$ III) were graded by the Clavien-Dindo classification.

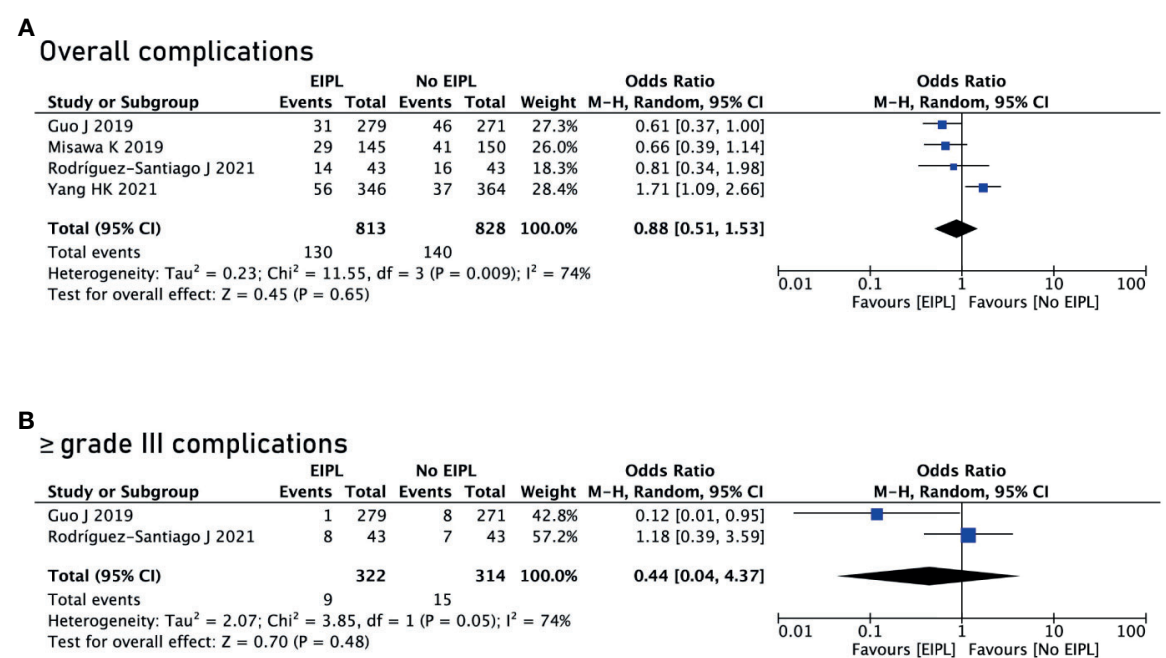

FIGURE 3 | Comparison of complications between EIPL group and No EIPL group. (A) Overall complications. (B) $\geq$ grade III complications. EIPL, extended intraoperative peritoneal lavage.

EIPL group in terms of OS $(\mathrm{HR}=0.77,95 \% \mathrm{CI}=0.36$ to 1.64 , $\mathrm{P}=0.49)$ (Figure 4A), $\mathrm{DFS}(\mathrm{HR}=0.97,95 \% \mathrm{CI}=0.71$ to $1.33, \mathrm{P}=$ 0.87 ) (Figure 5A), and PRFS ( $\mathrm{HR}=1.03,95 \% \mathrm{CI}=0.74$ to $1.43, \mathrm{P}=$ 0.86) (Figure 5B). One of the five RCTs included intraperitoneal chemotherapy (IPC) (17); therefore, we did a subgroup analysis of OS between EIPL group and No EIPL group. Furthermore, after pooling the data, the heterogeneity was decreasing obviously, and no difference was found between EIPL group and No EIPL group $(\mathrm{HR}=1.05,95 \% \mathrm{CI}=0.82$ to $1.34, \mathrm{P}=0.69)($ Figure $4 \mathbf{B})$.

\section{DISCUSSION}

A total of five RCTs with 1,790 patients were included in the current meta-analysis. No difference was found in baseline information. After pooling up the data of overall postoperative complications, no significant difference was found between EIPL group and No EIPL group. Furthermore, there was no significant difference between EIPL group and No EIPL group in terms of OS, DFS, and PRFS. 
A

Overall survival

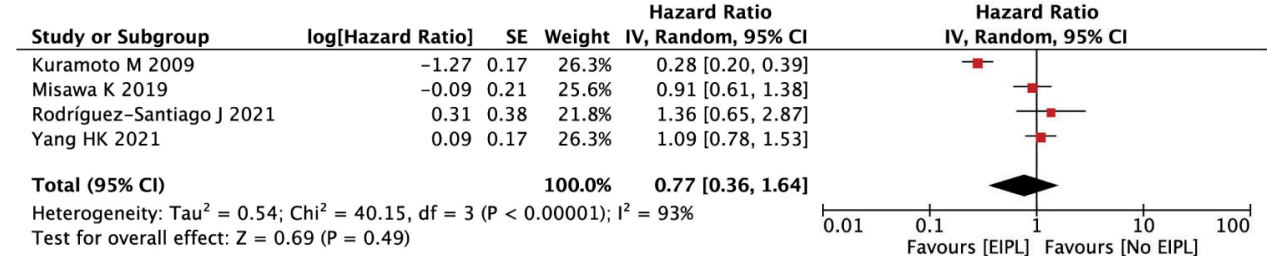

B

Subgroup analysis of overall survival

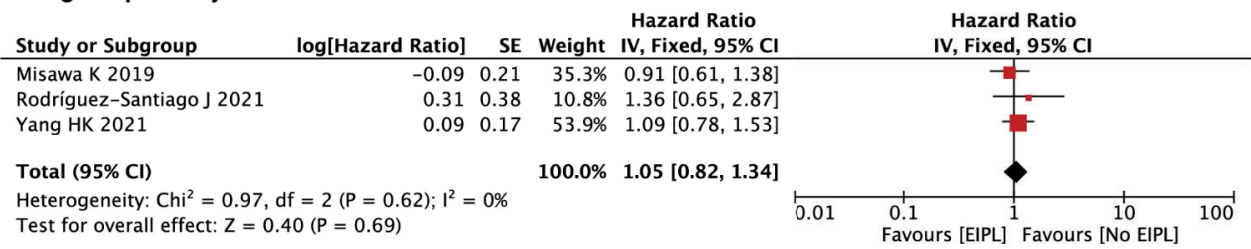

FIGURE 4 | Overall survival analysis between EIPL group and No EIPL group. (A) Overall survival. (B) Subgroup analysis of overall survival. EIPL, extended intraoperative peritoneal lavage.

A

Disease free survival

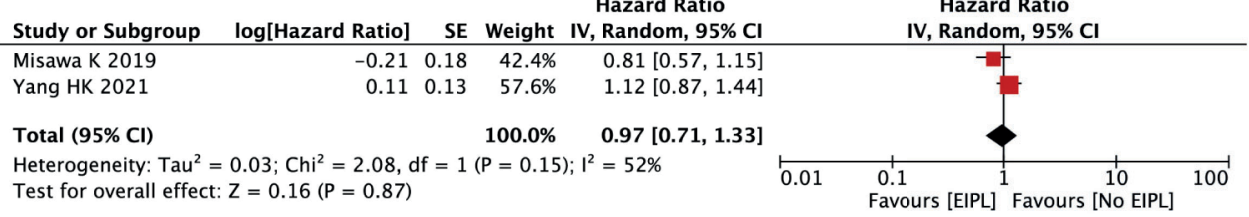

B

Peritoneal recurrence free survival

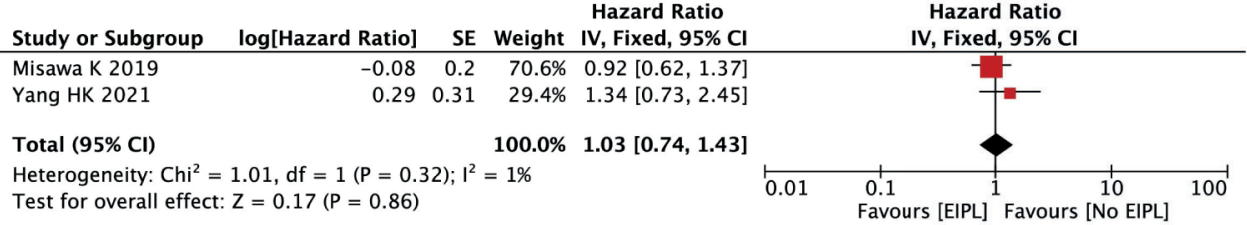

FIGURE 5 | Disease-free survival and peritoneal recurrence-free survival between EIPL group and No EIPL group. (A) Disease-free survival. (B) Peritoneal recurrence-free survival. EIPL, extended intraoperative peritoneal lavage.

EIPL and IPC are immediate treatment during surgery. Some studies reported that IPC was effective and that IPC could bring some benefits $(26,27)$. However, others considered that IPC had a negative effect (17). Furthermore, hyperthermic intra-operative peritoneal chemotherapy (HIPEC) was thought to be another treatment for improving OS and DFS after gastrectomy (28). EIPL is also a currently controversial method. Previous studies reported that EIPL could prolong OS and reduce complications $(16,17)$. However, other studies have shown that EIPL does not bring significant survival benefits and complications (18-20). Therefore, it is necessary to analyze the exact efficacy of EIPL.
Guo J et al. (16) reported that EIPL could reduce the incidence of postoperative complications including intra-abdominal abscesses, surgical infection, and postoperative death. The probable mechanism was that EIPL was performed 10 times with $1 \mathrm{~L}$ of saline, and the dilution method could greatly reduce the amount of damaged tissues and wound exudates in the peritoneum and clean the peritoneal cavity. However, another study found that the EIPL group had higher adverse events (19). It might be related to bowel manipulation during lavage and higher number of superficial wound infections in the EIPL group. In this meta-analysis, we found that there was no difference in postoperative complications 
between the EIPL and No EIPL groups, and furthermore, there was no difference in severe complications. Therefore, EIPL might potentially clean the abdominal cavity, but it did not reduce the incidence of complications.

A large amount of saline was used immediately during the operation, which could theoretically reduce the metastasis of free cancer cells in the peritoneum and abdominal cavity $(12,13)$. Kuramoto $M$ et al. (17) reported that the EIPL group improved OS compared to the No EIPL group. However, this study only included a small sample of 80 patients, and furthermore, the intervention group included IPC; therefore, the benefit of EIPL as a stand-alone therapy was still unknown. In this meta-analysis, after pooling up all the survival data, we found that there was no difference in terms of OS, and there was no difference between RFS or PRFS as well. It could be seen that EIPL had not achieved the expected results. However, there were only two research reporting RFS and PRFS, and the results might not be robust.

In addition to the overall postoperative complications and survival analysis, some studies reported some interesting clinical findings. Guo J et al. (16) reported that EIPL can significantly reduce postoperative pain, which might also be accidental. The probable reason was that the cause of pain reduction might be related to the reduction of inflammatory response. Pain is usually triggered by the local release of cytokines from inflammatory cells, which is a clinical reflection of tissue damage and inflammatory reactions $(16,29)$. Therefore, although EIPL was an interesting and simple intraoperative procedure, the current evidence could not support its widespread clinical application.

There were some certain limitations in the current metaanalysis. First, only five studies were included, which was

\section{REFERENCES}

1. Bray F, Ferlay J, Soerjomataram I, Siegel RL, Torre LA, Jemal A. Global Cancer Statistics 2018: GLOBOCAN Estimates of Incidence and Mortality Worldwide for 36 Cancers in 185 Countries. CA Cancer J Clin (2018) 68 (6):394-424. doi: 10.3322/caac.21492

2. Peng D, Cheng YX, Tao W, Zou YY, Qian K, Zhang W. Onco-Metabolic Surgery: A Combined Approach to Gastric Cancer and Hypertension. Cancer Manag Res (2020) 12:7867-73. doi: 10.2147/CMAR.S260147

3. Japanese Gastric Cancer Association. Japanese Gastric Cancer Treatment Guidelines 2014 (Ver. 4). Gastric Cancer (2017) 20(1):1-19. doi: 10.1007/ s10120-016-0622-4

4. Peng D, Cheng YX, Liao G. Effect of Endoscopic Resection on Short-Term Surgical Outcomes of Subsequent Laparoscopic Gastrectomy: A MetaAnalysis. World J Surg Oncol (2021) 19(1):119. doi: 10.1186/s12957-02102230-5

5. Kim SJ, Kim HH, Kim YH, Hwang SH, Lee HS, Park DJ, et al. Peritoneal Metastasis: Detection With 16- or 64-Detector Row CT in Patients Undergoing Surgery for Gastric Cancer. Radiology (2009) 253(2):407-15. doi: 10.1148/radiol.2532082272

6. Nakayama I, Chin K, Matsushima T, Takahari D, Ogura M, Shinozaki E, et al. Retrospective Comparison of S-1 Plus Cisplatin Versus S-1 Monotherapy for the Treatment of Advanced Gastric Cancer Patients With Positive Peritoneal Cytology But Without Gross Peritoneal Metastasis. Int J Clin Oncol (2017) 22 (6):1060-8. doi: 10.1007/s10147-017-1164-4

7. Yonemura Y, Endou Y, Shinbo M, Sasaki T, Hirano M, Mizumoto A, et al. Safety and Efficacy of Bidirectional Chemotherapy for Treatment of Patients With Peritoneal Dissemination From Gastric Cancer: Selection for relatively small; therefore, the results were not robust, and larger studies are needed. Second, DFS was chosen to be the primary outcome of the meta-analysis; however, peritoneal recurrence was difficult to detect from the included studies. Third, four RCTS were from Asian countries and one RCT was from western countries; the results might apply to Asian areas, and multicenter, multiregional high-quality RCTs should be carried out in the future.

In conclusion, EIPL did not bring benefit in terms of shortterm outcomes or survival. Therefore, EIPL is not recommended for patients undergoing curative gastrectomy for gastric cancer.

\section{DATA AVAILABILITY STATEMENT}

The original contributions presented in the study are included in the article/supplementary material. Further inquiries can be directed to the corresponding author.

\section{AUTHOR CONTRIBUTIONS}

DP and WT contributed to conception and design of the study. $\mathrm{X}$-YL organized the database. DP performed the statistical analysis. DP and WT wrote the first draft of the manuscript. Y-XC, HZ, CY, BZ, and BK wrote sections of the manuscript. All authors contributed to article and approved the submitted version.
Cytoreductive Surgery. J Surg Oncol (2009) 100(4):311-6. doi: 10.1002/ jso. 21324

8. Lei Z, Wang J, Li Z, Li B, Luo J, Wang X, et al. Hyperthermic Intraperitoneal Chemotherapy for Gastric Cancer With Peritoneal Metastasis: A Multicenter Propensity Score-Matched Cohort Study. Chin J Cancer Res (2020) 32 (6):794-803. doi: $10.21147 /$ j.issn.1000-9604.2020.06.12

9. Geng X, Liu H, Lin T, Hu Y, Chen H, Zhao L, et al. Survival Benefit of Gastrectomy for Gastric Cancer With Peritoneal Carcinomatosis: A Propensity Score-Matched Analysis. Cancer Med (2016) 5(10):2781-91. doi: $10.1002 / \mathrm{cam} 4.877$

10. Boku T, Nakane Y, Minoura T, Takada H, Yamamura M, Hioki K, et al. Prognostic Significance of Serosal Invasion and Free Intraperitoneal Cancer Cells in Gastric Cancer. Br J Surg (1990) 77(4):436-9. doi: 10.1002/ bjs. 1800770425

11. Kodera Y, Yamamura Y, Shimizu Y, Torii A, Hirai T, Yasui K, et al. Peritoneal Washing Cytology: Prognostic Value of Positive Findings in Patients With Gastric Carcinoma Undergoing a Potentially Curative Resection. J Surg Oncol (1999) 72(2):60-4; discussion 64-5. doi: 10.1002/(sici)1096-9098(199910) 72:2<60::aid-jso3>3.0.co;2-1

12. Yu XF, Ren ZG, Xue YW, Song HT, Wei YZ, Li CM. D2 Lymphadenectomy can Disseminate Tumor Cells Into Peritoneal Cavity in Patients With Advanced Gastric Cancer. Neoplasma (2013) 60(2):174-81. doi: 10.4149/neo_2013_023

13. Han TS, Kong SH, Lee HJ, Ahn HS, Hur K, Yu J, et al. Dissemination of Free Cancer Cells From the Gastric Lumen and From Perigastric Lymphovascular Pedicles During Radical Gastric Cancer Surgery. Ann Surg Oncol (2011) 18 (10):2818-25. doi: 10.1245/s10434-011-1620-8

14. Shimada S, Tanaka E, Marutsuka T, Honmyo U, Tokunaga H, Yagi Y, et al. Extensive Intraoperative Peritoneal Lavage and Chemotherapy for Gastric 
Cancer Patients With Peritoneal Free Cancer Cells. Gastric Cancer (2002) 5 (3):168-72. doi: 10.1007/s101200200029

15. Mayer AD, McMahon MJ, Corfield AP, Cooper MJ, Williamson RC, Dickson AP, et al. Controlled Clinical Trial of Peritoneal Lavage for the Treatment of Severe Acute Pancreatitis. N Engl J Med (1985) 312(7):399-404. doi: 10.1056/ NEJM198502143120703

16. Guo J, Xu A, Sun X, Zhao X, Xia Y, Rao H, et al. Combined Surgery and Extensive Intraoperative Peritoneal Lavage vs Surgery Alone for Treatment of Locally Advanced Gastric Cancer: The SEIPLUS Randomized Clinical Trial. JAMA Surg (2019) 154(7):610-6. doi: 10.1001/jamasurg.2019.0153

17. Kuramoto M, Shimada S, Ikeshima S, Matsuo A, Yagi Y, Matsuda M, et al. Extensive Intraoperative Peritoneal Lavage as a Standard Prophylactic Strategy for Peritoneal Recurrence in Patients With Gastric Carcinoma. Ann Surg (2009) 250(2):242-6. doi: 10.1097/SLA.0b013e3181b0c80e

18. Misawa K, Mochizuki Y, Sakai M, Teramoto H, Morimoto D, Nakayama H, et al. Randomized Clinical Trial of Extensive Intraoperative Peritoneal Lavage Versus Standard Treatment for Resectable Advanced Gastric Cancer (CCOG 1102 Trial). Br J Surg (2019) 106(12):1602-10. doi: 10.1002/bjs.11303

19. Yang HK, Ji J, Han SU, Terashima M, Li G, Kim HH, et al. Extensive Peritoneal Lavage With Saline After Curative Gastrectomy for Gastric Cancer (EXPEL): A Multicentre Randomised Controlled Trial. Lancet Gastroenterol Hepatol (2021) 6(2):120-7. doi: 10.1016/S2468-1253(20)30315-0

20. Rodríguez-Santiago J, Luna A, Garsot E, Aldeano A, Balagué C, Rada A. Extended Intraoperative Peritoneal Lavage as Prophylactic Peritoneal Recurrence for Locally Advanced Gastric Cancer: A Prospective Randomized Trial. Clin Transl Oncol (2021) 23(9):1857-65. doi: 10.1007/ s12094-021-02596-8

21. Moher D, Liberati A, Tetzlaff J, Altman DGPRISMA Group. Preferred Reporting Items for Systematic Reviews and Meta-Analyses: The PRISMA Statement. PloS Med (2009) 6(7):e1000097. doi: 10.1371/journal.pmed. 1000097

22. Dindo D, Demartines N, Clavien PA. Classification of Surgical Complications: A New Proposal With Evaluation in a Cohort of 6336 Patients and Results of a Survey. Ann Surg (2004) 240(2):205-13. doi: 10.1097/01.sla.0000133083. 54934.ae

23. Higgins JP, Altman DG, Gøtzsche PC, Jüni P, Moher D, Oxman AD, et al. The Cochrane Collaboration's Tool for Assessing Risk of Bias in Randomised Trials. BMJ (2011) 343:d5928. doi: 10.1136/bmj.d5928
24. Ioannidis JP. Interpretation of Tests of Heterogeneity and Bias in Meta-Analysis J Eval Clin Pract (2008) 14(5):951-7. doi: 10.1111/j.1365-2753.2008.00986.x

25. Higgins JP, Thompson SG, Deeks JJ, Altman DG. Measuring Inconsistency in Meta-Analyses. BMJ (2003) 327(7414):557-60. doi: 10.1136/bmj.327.7414.557

26. Tsujitani S, Okuyama T, Watanabe A, Abe Y, Maehara Y, Sugimachi K. Intraperitoneal Cisplatin During Surgery for Gastric Cancer and Peritoneal Seeding. Anticancer Res (1993) 13(5C):1831-4.

27. Yang $\mathrm{Y}, \mathrm{Li} \mathrm{Y}, \mathrm{Du} \mathrm{X}$. Does Intraperitoneal Chemotherapy Increase the Incidence of Anastomotic Leakage After Colorectal Cancer Surgery: A Systematic Review and Meta-Analysis. Gastroenterol Res Pract (2021) 2021:9204373. doi: 10.1155/2021/9204373

28. Granieri S, Bonomi A, Frassini S, Chierici AP, Bruno F, Paleino S, et al. Prognostic Impact of Cytoreductive Surgery (CRS) With Hyperthermic Intraperitoneal Chemotherapy (HIPEC) in Gastric Cancer Patients: A Meta-Analysis of Randomized Controlled Trials. Eur J Surg Oncol (2021) S0748-7983(21):00492-3. doi: 10.1016/j.ejso.2021.05.016

29. Ruiz-Tovar J, Llavero C, Muñoz JL, Zubiaga L, Diez M. Effect of Peritoneal Lavage With Clindamycin-Gentamicin Solution on Post-Operative Pain and Analytic Acute-Phase Reactants After Laparoscopic Sleeve Gastrectomy. Surg Infect (Larchmt) (2016) 17(3):357-62. doi: 10.1089/sur.2015.196

Conflict of Interest: The authors declare that the research was conducted in the absence of any commercial or financial relationships that could be construed as a potential conflict of interest.

Publisher's Note: All claims expressed in this article are solely those of the authors and do not necessarily represent those of their affiliated organizations, or those of the publisher, the editors and the reviewers. Any product that may be evaluated in this article, or claim that may be made by its manufacturer, is not guaranteed or endorsed by the publisher.

Copyright (c) 2021 Tao, Liu, Cheng, Kang, Zhang, Yuan, Zhang and Peng. This is an open-access article distributed under the terms of the Creative Commons Attribution License (CC BY). The use, distribution or reproduction in other forums is permitted, provided the original author(s) and the copyright owner(s) are credited and that the original publication in this journal is cited, in accordance with accepted academic practice. No use, distribution or reproduction is permitted which does not comply with these terms. 